\title{
Biogeochemical Research with an Autonomous Underwater Vehicle: \\ Payload Structure and Arctic Operations
}

\author{
Thorben Wulff, Sascha Lehmenhecker, Dr. Eduard Bauerfeind, Ulrich Hoge \\ Department for Deep Sea Ecology and Technology \\ Alfred Wegener Institute for Polar and Marine Research \\ 27570 Bremerhaven, Germany \\ Thorben.wulff@awi.de
}

\author{
Kimberly Shurn \\ Marine Operations Engineering \\ Bluefin Robotics Corporation \\ Quincy, Massachusetts 02169, USA \\ kshurn@bluefinrobotics.com
}

\author{
Dr. Michael Klages \\ Sven Lovén Center for Marine Sciences \\ University of Gothenburg \\ 45178 Fiskebäckskil, Sweden \\ michael.klages@gu.se
}

\begin{abstract}
In modern polar marine research, Autonomous Underwater Vehicles (AUV) gain increasing importance due to their ability to take on high risk or time-consuming tasks in the harsh physical environment of the polar seas. They represent a relatively new group of robots that can investigate large areas in all three dimensions without the direct need of human surveillance. The most common objective for these vehicles that are currently operated worldwide is to gather sonar data and create highresolution maps of the sea floor. A relatively new task is their deployment within the framework of water column studies dealing with the investigation of biogeochemical processes in the open sea or in ice-covered areas.
\end{abstract}

The Alfred Wegener Institute for Polar and Marine Research (AWI) in Bremerhaven, Germany operates a 21-inch class AUV by the American manufacturer Bluefin Robotics (Quincy, Massachusetts) named PAUL. The main objectives of the herein described AUV project at AWI are the investigation of biogeochemical processes in the surface water and to analyze the stratification of the upper water column in the marginal ice zone as well as the dynamic interaction between ice and ocean. For this reason, since 2008, PAUL has been equipped with a number of biogeochemical sensors (e.g. Nitrate, Oxygen, Fluorescence, etc.) and a newly designed water sample collector all specified for operations in icy waters.

In the course of several Arctic expeditions onboard the RV Polarstern, the vehicle was deployed in the Fram Strait close to the AWI deep sea observatory "HAUSGARTEN". In a first series of diving trials in ice covered areas, the first mission under ice accomplished by PAUL occurred at the HAUSGARTEN site in summer 2010.

Here, the description of the vehicle, the mode of operations and especially the structure of the payload is detailed. The payload development process took advantage of complementary instruments: combining data of different sensors with water samples. The samples allow data to be obtained for parameters that cannot be measured in situ, for example the amount of microscopic plankton, and in addition, they can also be used for validation purposes. For example, by measuring the amount of chlorophyll a in distinct samples, the flow-through chlorophyll a fluorometer of the AUV can be calibrated. This provides more accurate values than a calibration with laboratory standards. Results of such measurements gathered from 2010 to 2012 (ARK 25/2 - ARK 27/2, RV Polarstern) are presented exemplarily for the scientific use of the vehicle.

Additionally, a description will be given for the new technologies that were developed (remotely operated flying drones, GPS tracking of the ice edge) the new procedures (vehicle tracking, special dive maneuvers) introduced into the AUV project in order to acquire a comprehensive picture of the physical and biogeochemical conditions of the pelagic environment assessed. These steps were mandatory, not only to ensure the safety of the vehicle, but also to be able to place the measured data into the greater context of challenges in understanding the dynamics of icecovered seas. 


\section{INTRODUCTION}

The Alfred Wegener Institute for Polar and Marine Research (AWI) in Bremerhaven, Germany is one of three institutes in Germany that operates an Autonomous Underwater Vehicle (AUV) to pursue scientific objectives. The Center for Marine Environmental Sciences of the University of Bremen (MARUM) and the GEOMAR Helmholtz Center for Ocean Research in Kiel are the other institutes that also operate AUVs for scientific reasons. The general goals of these institutions differ - with the AWI focusing on investigations in the Polar Regions. With the particular goal of operations in high latitudes, a 21-inch class AUV from the American manufacturer Bluefin Robotics (Quincy, Massachusetts) was acquired in 2003 and named PAUL. Since 2008, the entire AUV project at AWI has focused on the investigation of the upper water column, the micro layers in the marginal ice zone, and the analysis of biogeochemical processes close to the surface.

The surface water layers of the oceans differ from the deeper water masses by the abundance of light and the gas exchange with the earth's atmosphere. As a consequence, photosynthetic primary production is exclusively tied to the upper water column. Organic particles that have been formed at the surface sink to the sea floor and then serve as an energy source for benthic life; thus connecting the upper and deeper water layers. With a certain time delay, surface bound processes are reflected on the sea floor. In the polar oceans, the ice coverage represents a unique variable that influences environmental conditions from sea surface to the bottom of the ocean. The low water temperatures of the polar oceans support the solubility of gases, but with a solid ice cover on top of the water, the gas exchange with the atmosphere is reduced to almost zero. Ice reduces the transmission of light and thus decreases primary production rates. However, at the same time, the stable summer stratification of the surface water, a direct result of the melting of ice, is the reason for the high biological productivity of the marginal ice zone. The stratification and the consequences have already been investigated in the 1980's for example by Smith et al. in 1984 [1] or Niebauer et al. in 1989 [2]. These kinds of effects, which are partly in competition to each other, make the Arctic Ocean a spatially and temporarily dynamic environment. Additionally, questions as to stratification and productivity of ice-covered seas gain even more importance against the background of climate change and a fast changing Arctic. The most visible consequence of the climate change is the dramatic sea ice retreat that could be observed in the last decades. The loss of sea ice causes fundamental changes in the Arctic habitat as it was described for example by Arrigo et al. in 2008 [3]. AUVs offer the possibility to observe these processes in a very efficient way. Travelling at relatively high speeds, these vehicles can collect data from various locations within a short matter of time, thus providing an almost synoptic, three dimensional picture of the environment [4]. As there is no direct physical connection to a support vessel, these vehicles can also operate in regions almost inaccessible by traditional methods. As a consequence, one of the key requirements is reliable navigation of these vehicles under ice. Although there have been a number of attempts, with some groups achieving remarkable success, for example the Autosub missions of the National Oceanography Center in Southampton in 2009 [5], these kind of missions still remain a challenge.

Since 2009, PAUL has accomplished four cruises into the Arctic. In order to study the biogeochemical interrelations in the marginal ice zone, five still relatively short missions were conducted below the ice. All Arctic missions were conducted in the Fram Strait between Svalbard and Greenland. This region is of high scientific interest as this strait represents the only deep water connection between the Arctic basin and the Earth's oceans [6]. Starting in 1999, AWI has managed its deep sea observatory HAUSGARTEN in this area and the AUV project at AWI aims at connecting surface water data to benthic data which are regularly gathered at the HAUSGARTEN site.

\section{THE VEHICLE}

A 21-inch class AUV from the American manufacturer Bluefin Robotics serves as a carrier vehicle (Fig. 1). This class of torpedo-shaped vehicles is rated to a maximum depth of 3000 meters (the latest model has a 4500 meters depth rating) and has an outer diameter of 53.3 centimeters $(=21$ inches $)$. The vehicles are divided into sections that are exchangeable according to their respective mission. Due to this modular design and desired payload, the length of a vehicle varies. In the current configuration, PAUL has an overall length of 4.3 meters. The tail section contains the Main Vehicle Computer (MVC), communication and tracking systems, and the thruster. The thruster is a single gimballed ducted thruster that moves horizontally and vertically with the aid of linear drives. Apart from the thruster, the vehicle does not have any other control equipment such as rudders that have the potential to be damaged

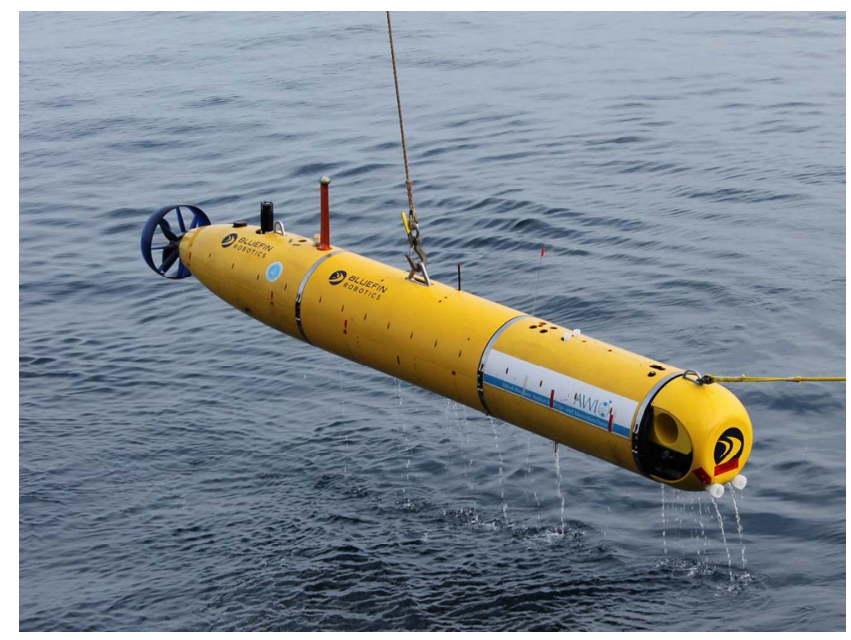

Fig. 1: PAUL is being recovered. 
if the vehicle surfaced between ice floes. The centre section of the vehicle houses the batteries. This particular section currently contains three batteries with a possibility to use a maximum of six batteries. Each battery has a capacity of 1.5 kilowatt hours allowing the vehicle to travel a distance of approximately 70 kilometers. Additionally, the battery section also provides the central single lift point of the vehicle and the emergency ascent drop weight is installed here. The vehicle's payload section is located forward of the battery section and is described in detail in this paper. The foremost section is the nose section containing a jettison abled float with an attached recovery line.

The vehicle's navigation system is a Kearfott Inertial Navigation System (INS) of the type KN-5053 and is aided by the following sensors:

- $\quad$ Teledyne RDI: Workhorse Navigator Doppler Velocity Log (DVL) with 300 kilohertz.

- Paroscientific Inc.: Digiquartz pressure sensor (4000 meters).

- Thales Navigation: Ashtech DG-14 GPS receiver.

- Sea Bird Electronics SBE 49 FastCat Conductivity, Temperature and Depth probe is used to provide an insitu sound profile to support DVL measurements.

The outer hull of the AUV forms a hydrodynamic efficient housing, and also allows for each section to be free flooded. Consequently, every scientific instrument integrated in the payload section is directly exposed to environmental conditions like pressure and cold temperatures. Thus, every instrument has to be protected by an individual pressure hull. The weight of these pressure hulls can sum up to a large part of the payload's total weight - reducing the valuable interior volume of the vehicle as this weight has to be compensated by buoyancy modules. With respect to the objectives of the project, namely investigating the upper water column, the maximum operation depth of the payload was defined to be at 600 meters. This limitation made it possible to use lighter instruments with thinner pressure hull walls and less dense (= more efficient) buoyancy foam as the foam does not have to withstand high pressure. The 600 meter depth rating is merely valid for the current and herein described payload section. As the vehicle consists of different sections that can easily be exchanged, the rest of the vehicle remained equipped with 3000 meter rated buoyancy modules. Thus, after a quick reconfiguration that only involves the payload section, the vehicle is able to cover its full operational range again.

\section{THE PAYLOAD SECTION}

\section{A. Sensors}

The number of sensors needed to capture every biogeochemical parameter fairly exceeds the vehicle's payload capacity. Therefore it is necessary to define certain expressive parameters and to integrate the respective sensors. The current integrated instruments and the measureable parameters are as follows:

- Nitrate

Satlantic SUNA deep Nitrate sensor

The amount of nitrate in the water is measured by the absorption of ultraviolet (UV) light (190 - 370 nanometers) following the basic principle of Johnson and Coletti [7].

- Dissolved Carbon Dioxide $\left(\mathrm{CO}_{2}\right)$ Contros HydroC $\mathrm{CO}_{2}$ Sensor

Using a semi permeable membrane, $\mathrm{CO}_{2}$ molecules are extracted from the sea water and channeled into a measuring chamber. Here the amount of $\mathrm{CO}_{2}$ is determined using a Nondispersive Infrared Sensor (NDIR).

- $\quad$ Dissolved Oxygen (DO)

Sea Bird SBE 43 (Version: SBE 43-2a)

The sensor's functional principle is based on a Clark Electrode. In order to minimize the response time a profiling configuration sensor (membrane thickness: 0.5 millimeters) is used.

- $\quad$ Conductivity, Temperature and Depth probe (CTD) Sea Bird SBE 49 FastCat (Version: SBE 49-1f)

The CTD is both a part of the scientific payload and of the vehicle navigation system. The SBE 49 was chosen as it has a short response time and is thus suitable for fast moving platforms like an AUV.

- Colored Dissolved Organic Matter (CDOM)

Turner Designs C7 "U”

For CDOM this fluorometer works with an excitation wavelength of 325 nanometers and a detected emission wavelength of 470 nanometers.

- Clorophyll a (Chl. a)

Turner Designs C7 "C"

For chlorophyll a, this fluorometer works with an excitation wavelength of 465 nanometers and a detected emission wavelength of 696 nanometers.

- Photosynthetically Active Radiation (PAR)

Satlantic PAR Sensor (Version: PAR-log-s)

As the AUV project includes missions below sea ice a logarithmic sensor is integrated in the vehicle to make use of its high resolution capabilities at low PAR values.

Data recording and power supply is centrally managed by the vehicle's Payload Control Computer (PCC) which was specifically designed for that purpose. The PCC is based on a VIA Pico ITX mainboard equipped with a 1 gigahertz VIA C7 processor running on an optimized version of Microsoft Windows $\mathrm{XP}^{\circledR}$. Signals of the sensors are transmitted to the PCC in the form of analog signals. Data are digitalized by a 13 bit analog-digital converter and saved on a solid state hard disk. 


\section{B. Water Sample Collector}

As some parameters cannot be measured in situ yet, the vehicle carries a water sample collector. AUV based water sample collectors are rather special payload components and operated by just a small number of institutions. Apart from the AWI, the Monterey Bay Aquarium Research Institute (MBARI, Monterey, USA) developed a sample collector called GULPER for one of their vehicles [8]. The sample collector from AWI was an in-house development that was put into service in July 2009 (RV Polarstern). With the aid of the sample collector the vehicle is able to collect a maximum of 22 water samples with a total volume of 4.8 liters. The volume of each sample is 220 milliliters. To provide the sample collector with water, the forward movement of the vehicle and the resulting dynamic pressure in front of its nose is used. Funnels that stick out of the vehicle's nose channel the water into pipes and direct it into the vehicle's payload section. The actual sample containers work similar to Niskin bottles and are made of polyvinylidene fluoride (PVDF) to meet high purity standards of various biogeochemical analyses. The functional principle of the sample collector is described more detailed in [9].

\section{Data Fusion}

One of the primary objectives while designing the payload section was to make use of complementary instruments. A common example for data fusion is the interaction between a CTD and a DO sensor. A Parameter such as salinity is derived from the CTD data and is necessary to calculate the oxygen content and the current oxygen saturation [10]. Also, by using CTD data, temperature dependent effects on the absorption spectrum of bromide ions, which absorb UV-light at a similar range as nitrate and thus interfere with the nitrate measurement, can be compensated ([11], [12]).

One "special" feature of PAUL is the possibility to combine sensor data with the data of simultaneously taken water samples. Apart of providing material for investigations that can only be carried out with samples (e.g. amount of micro plankton), the samples are also used for a ground truth and calibration purposes. During the two cruises HE-377 (RV Heincke, April 2012) and ARK 27/2 (RV Polarstern, July 2012), the following parameters have been determined using AUV water samples: Total Alkalinity (TA), Dissolved Inorganic Carbon (DIC), Inorganic Dissolved Nutrients (IDN) and Chlorophyll a (Chl a).

Derived from the TA and DIC values, the partial pressure of $\mathrm{CO}_{2}$ can be calculated following Zeebe and Wolf-Gladrow [13] and the $\mathrm{CO}_{2}$ sensor data can be verified by an independent source. As for the $\mathrm{CO}_{2}$ sensor, the nitrate value can also be verified using the samples. Within the framework of the IDN analysis, the amount of the four different nutrients ammonium, phosphate, silicate, and nitrate is determined by default. Thereby a ground truth for the nitrate sensor is done automatically.
Unlike the processes mentioned before, measuring the amount of chlorophyll a is not only important for signal verification but to calibrate the signals of the chlorophyll a fluorometer. As the fluorescence response of phytoplankton is not constant but varies with species [14] and due to the Kautsky effect [15], quantitative chlorophyll a measurements can only be achieved after an in-situ calibration of the instrument [16].

\section{Structural Set-up}

According to the torpedo shape of the vehicle the payload section is cylindrically shaped as well. The length of the section is 1015 millimeters and the inner diameter is 520 millimeters. The section can be separated into a lower and upper half shell. The water sample collector represents the largest single instrument in the payload section. It has some special requirements distinguishing it from the sensors. The most important difference is that the sample collector needs to be easily accessible as the samples have to be processed in the ship's laboratories as soon as possible after the vehicle has been recovered. Additionally, the sample collector is passively flushed - using only the dynamic pressure in front of the vehicle as it cruises. In order to optimize the flow rate in the supply pipes, it is an asset to keep the pipes as short as possible meaning the position of the sample collector needed to be as close to the vehicle's front end as possible. These two basic requirements of the sample collector, accessibility and position, were important boundary conditions for the further design work. The design of the payload section can be seen in Fig. 2 and Fig. 3. In front of the sample collector the DO sensor, the CDOM sensor and two supply pumps are positioned. Located above the sample collector, the $\mathrm{CO}_{2}$ and the nitrate sensor are both supplied with water via a shared water circuit for their integration. In addition to that, the PAR sensor is positioned above the sample collector in an upward looking configuration. Behind the sample collector, the chlorophyll a fluorometer and the PCC are integrated.

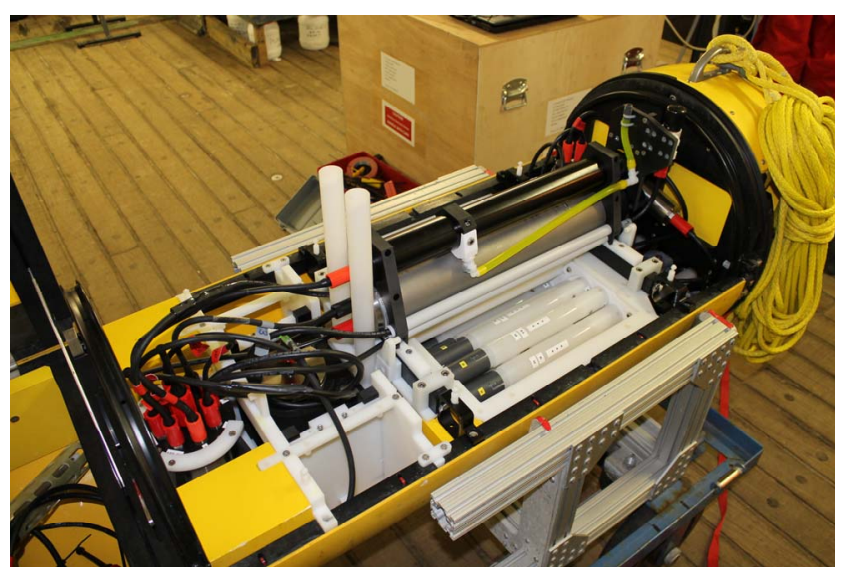

Fig. 2: Payload section during ARK 27/2 prior to a dive. The sample containers are already installed. 


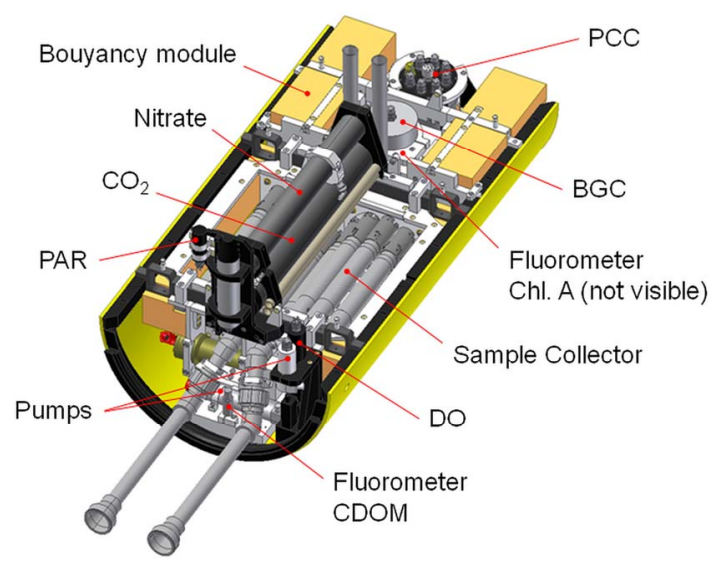

Fig. 3: Computer-aided design model of the payload section.

To be able to integrate instruments on short notice, for example instruments that come from external partners, the payload section has an exchangeable module. This module consists of a plastic box which can be inserted into the payload section like a drawer. Below the module the outer skin of the vehicle has an opening to provide instruments with a direct access to the water. The module has a base area of $189 \times 153$ millimeters; the available volume is approximately 7.3 liters. The first external user instrument which took advantage of this possibility was the BioGeoChemical Module (BGC) of the Max Planck Institute for Marine Microbiology in Bremen, Germany, during two cruises in 2012.

All structural parts of the payload section consist of lightweight polyethylene (PE). With an average density of 0.98 gram per cubic centimeter this material contributes to the vehicle's overall buoyancy. The actual buoyancy modules are made of microballoon based synthetic foams of the type TG-24 manufactured by Trelleborg Offshore, UK. The buoyancy modules have an average density of 0.385 gram per cubic centimeter and are approved for a maximum water depth of 2000 meters. This depth rating largely covers our field of interest (maximum 600 meter depth) but at the same time it allows us to extend the mission depth in the future. The currently available buoyancy reserve within the payload section is approximately 160 newtons.

\section{ICE AND VEHICLE TRACKING}

Currently, unattended AUV missions in the open water column, particularly below ice are considered to be high risk. Additionally, navigation errors, which inevitably occur during missions in the open water column, can only be corrected when the vehicle is constantly tracked. When diving under ice, tracking the ice drift and the ice edge's orientation are also critical issues - not just to ensure the safety of the vehicle but also to support the scientific interpretation of the AUV data.
To track the vehicle under water the Ultra Short Baseline (USBL) system "GAPS" of the French manufacturer iXBlue (Marly le Roi, France) is used. According to manufacturer information the maximum range of this system is 4000 meters in all directions [17]. Considering the particular objective to conduct shallow missions in the upper water column, it is of particular interest that GAPS covers an operating field of $200^{\circ}$ [17]. Thus it is possible to track objects close to the surface although the transducer is positioned underneath a ship.

Tracking of the ice is done by Global Positioning System (GPS) based tracking units which are deployed on the ice. These units determine their own position using GPS and transmit it via radio communication. By deploying at least two of these units it is possible to observe the ice edge's orientation. In 2012, during ARK 27/2, a remotely controlled flying drone was deployed on the ice for the first time to send position updates and collect PAR data [18].

All tracking signals are centrally displayed on the operators screen. Aside of the AUV and the ice, the ship itself and the boat which is used for recovery are put on display as well. Thus the operator can easily overlook the entire mission and can for example guide the recovery team to the vehicle in case of foggy conditions. Additionally the operators screen is broadcasted onto the bridge of the ship - making the communication with the ship's command safe and easy.

\section{FloAT MANEUVER AND DATA CORRECTION}

\section{A. Float maneuver}

At the beginning of 2012 a new maneuver was introduced into AWI's AUV project in order to investigate the micro stratification of the upper water column: The "Float"-maneuver. This maneuver takes advantage of the approximately 30 newtons residual buoyancy of the vehicle. As the vehicle reaches a specific position it stops the thruster and starts a slow ascent towards the surface, allowing the sensors to record a detailed vertical cast of the respective location. When the vehicle reaches a specific depth it reactivates the thruster and descends again to transit to the next Float location. Several Float maneuvers in a particular area can provide a detailed three dimensional picture of the investigated water volume. The first tests of the Float maneuver were conducted in March 2012 at the Bluefin Robotics test site onboard the RV Resolution. The maneuver was further analyzed during the cruise HE-377 in the North Sea in April 2012 (RV Heincke). First scientific operations in polar waters and under ice were accomplished in July 2012 during expedition ARK 27/2 in the Fram Strait (RV Polarstern).

The Float maneuver combines three major advantages:

a) As the vehicle ascends slowly, it achieves a fine resolution of the water column. PAUL's typical ascent velocities are between 10 and 20 centimeters per second. As the data recording 
frequency is 1 hertz, the vertical resolution is 10 to 20 centimeters as well.

b) As RV Polarstern is an icebreaker, she has a heavy hull and a relatively large draft of approximately 12 meters. Thus, at least to this depth, one can assume that the fine stratification of the uppermost water column is destroyed and data derived from ship bound measurements do not necessarily represent correct environmental conditions. In contrast to that, PAUL crosses the water layers smoothly and causes as few disturbances as possible.

c) Due to the higher risk for the vehicle, missions below the ice are conducted within the GAPS tracking range. As the vehicle ascends almost vertically during the Floats, the distance to the support ship remains almost the same. Thus, compared to an undulating manner of driving, a bigger number of vertical casts can be recorded without leaving the GAPS tracking range.

\section{B. Data Correction}

Initially the term "micro layers" is not related to a specific size scale. In 1993 Cowles and Desiderio emphasized the need for sub-1-meter resolution and ultimately achieved a vertical resolution of 2 centimeters with their Rapid Sampling Vertical Profiler (RSVP) [19]. Using an AUV (and other instruments) and applying the three criteria defined by Dekshenieks et al. in [20] to identify thin phytoplankton layers, Ryan et al. detected layers with a minimum thickness of 1 meter in the Monterey Bay in 2008 [21]. The scientific requirements defined PAUL to achieve a comparable resolution. In order to detect layers of that size and precisely correlate the measurement data of the different sensors, it is necessary to provide every parameter with an accurate depth stamp. As the depth sensor is positioned in the rear of the vehicle but the payload section is at the front end, for example a relatively small pitch angle can cause a significant depth error.

\section{a) Correction due to Pitch and Roll Angle}

To be able to calculate an exact depth stamp for every parameter, the position of the "sampled spot" relative to the vehicle's depth sensor has to be known. In the case of sensors that protrude out of the vehicle into the water, the sampled spot was defined to be the sensitive surface of the instrument. In the case of sensors that are connected to a pumped water circuit, the sampled spot is the position of the water inlet at the vehicle. Measuring these positions, with the depth sensor of the vehicle as the point of origin, result in individual position vectors for every parameter. Depending on the pitch and roll angle of the vehicle and using rotation matrices these vectors can be rotated into their real orientation.

Rotations in a three-dimensional space about an arbitrary axis can be described by the following matrix [22]:

$$
R_{\vec{n}}(\alpha)=\left(\begin{array}{ccc}
n_{x}^{2} t+r & n_{x} n_{y} t-n_{z} s & n_{x} n_{z} t+n_{y} s \\
n_{x} n_{y} t+n_{z} s & n_{y}^{2} t+r & n_{y} n_{z} t-n_{x} s \\
n_{x} n_{z} t-n_{y} s & n_{y} n_{z} t+n_{x} s & n_{z}^{2} t+r
\end{array}\right)
$$

Where $n_{x}, n_{y}, n_{z}$ represent the Cartesian components of the unit vector of the rotation axis $\vec{n}$.

With $\alpha$ being the angle of the rotation, the variables $r, s$ and $t$ stand for:

$$
\begin{gathered}
r=\cos \alpha \\
s=\sin \alpha \\
t=(1-\cos \alpha)
\end{gathered}
$$

As pitch and roll movements are two independent transformations with different angles and about different axes, two separate matrices have to be created. With the axes and angle convention given in Fig. 4 and may $\vec{u}$ represent the position vector of one sensor, $\vec{u}$ " this position vector in its real orientation and $\overrightarrow{x^{\prime}}$ the unit vector of the vehicle's longitudinal axis rotated by the pitch angle, this leads to the equation:

$$
\overrightarrow{u^{\prime \prime}}=\left(u_{x}^{\prime \prime}, u_{y}^{\prime \prime}, u_{z}^{\prime \prime}\right)^{T}=\left[R_{\overrightarrow{x^{\prime}}}(\phi) \cdot\left(R_{\vec{y}}(\theta) \cdot \vec{u}\right)\right]
$$

It is to be mentioned that the heading of the vehicle is disregarded. Thus the first rotation (pitch) is assumed to be around the global y-axis with its unit vector $\vec{y}=(0,1,0)^{T}$.

As it is only the vertical component of the rotated position vector $\vec{u}$ " which is necessary for correcting the depth, it follows:

$$
\text { depth }_{\mathrm{corr}}=\text { depth }_{\mathrm{raw}}+u_{\mathrm{z}}^{\prime \prime}
$$

Based on these equations each sensor has an individual depth stamp.

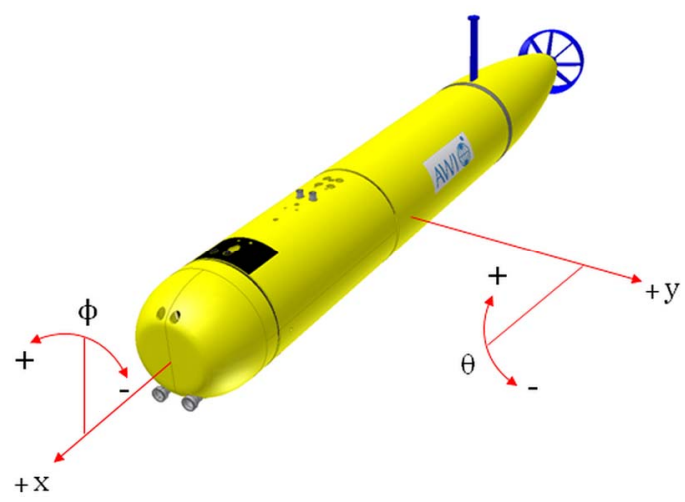

Fig. 4: Orientation of axes and angles used for depth correction. 


\section{b) Correction of the PAR data}

As the PAR measurement is an irradiance measurement and the PAR sensor is equipped with a cosine collector, the inclination of the sensor causes a shift in the data. According to Kirk [23], at a certain depth one can assume that most of the light in the water travels downwards. Thus, the inclination of the sensor with respect to the vertical is decisive for data correction. The real orientation of the sensor, represented by the normal vector of the sensor's sensitive surface $(\vec{p})$, can be calculated using rotation matrices like (1). The inclination angle can be derived from the scalar product of the vertical vector $\vec{z}$ and the direction vector of the PAR sensor $\vec{p}$. Within a tolerance limit of $5^{\circ}$ for the inclination angle following Sturesh et al. [24], this yields to:

$$
P A R_{\text {corr }}=\left(\frac{\vec{p} \cdot \vec{z}}{|\vec{p}| \cdot|\vec{z}|}\right)^{-1} \cdot P A R_{\text {raw }}
$$

\section{c) Correction due to Pump Time Delay}

Some sensors in the vehicle are operated with a flow cell and thus are connected to a pumped water circuit. Depending on the flow rate and the volume of the respective supply line, a certain delay occurs in the sensor signal. For this reason, flow meters of the type 300-010 manufactured by Titan Enterprises Ltd. are installed in the supply lines. Unfortunately these devices are neither watertight nor pressure resistant. However, using silicone, they can be sufficiently sealed to measure the flow rate at the surface. Although the flow meters' electronics is destroyed when the vehicle starts a deeper dive, the devices remain in the supply lines so that the previously determined flow rate is not changed. In the flow meters, the flow rate is measured using a free-running paddle wheel spinning on a sapphire axis. Revolutions of that wheel are counted by a magnetic encoder. Although the flow meter is used in conditions way beyond its specifications as soon as the vehicle dives and might face pressure induced stress, the assumption is, the flow meter does not cause any changes in the flow rate as the paddle wheel is mechanically decoupled.

Based on the volume of the respective supply line and the measured flow rate at surface conditions, the signal delay is calculated for every parameter.

\section{RESULTS}

Dividing the entire dive into separate mission phases has turned out to be advantageous. In the first phase the vehicle executes a number of Floats in a specific area to analyze the water column structure. In the second phase of the dive the vehicle dives down and starts taking water samples either in a constant depth or at different depth levels in ascending order. As the samples are collected during the last phase of the dive, they remain onboard the AUV for as short as possible before being processed and conserved in shipboard laboratories. When diving in the open water (no ice), the different phases of the dive are programmed within one single mission file.

In the case of dives under or close to the ice edge, the unpredictable ice drift poses a serious risk to the vehicle as the preprogrammed surface point could be covered by ice. The individual phases of the dive are thus separated in short mission files of 1-2 hours. After the vehicle completed a specific phase, it surfaces and allows the position, calculated by the INS, to be corrected via GPS and then waits for the next dive file. With these short missions the operator is able to adapt the dive plan according to the observed ice drift.

\section{A. Open Water}

Dive No. 3 of the expedition ARK 27/2 exemplarily represents one of PAUL's open water dives (Fig. 5). The red circles represent positions where the vehicle took water samples. Fig. 6 shows the raw signal of the chlorophyll a fluorometer and the sample derived amount of chlorophyll a, both represented versus mission time of dive No. 3. The fluorometer was subsequently calibrated using these data and additional data of one prior dive. The calibration curve is shown in Fig. 7. Based on this calibration, the chlorophyll a distribution during the Float phase of dive No. 3 can be seen in Fig. 8. Data like nitrate or dissolved oxygen can be presented accordingly (Fig. 9, Fig. 10) and show the expected correlations. Phytoplankton, indicated by the chlorophyll a value, produces oxygen and consumes nitrate as a nutrient. Thus, high chlorophyll a concentrations go along with low nitrate values and high dissolved oxygen values.

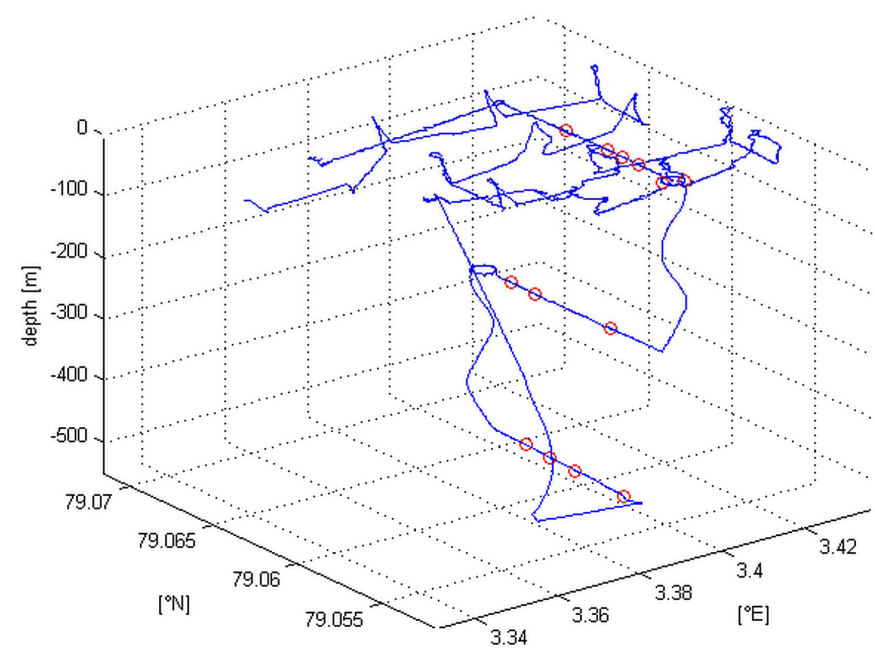

Fig. 5: Track of dive No. 3 (ARK 27/2). 


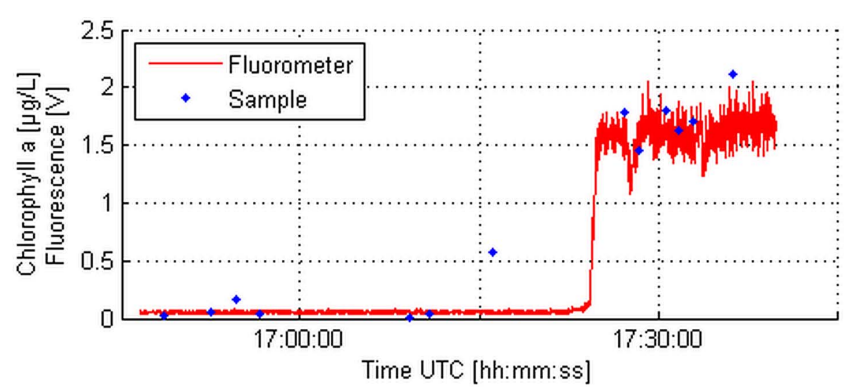

Fig. 6: Raw Fluorescence signal and sample derived chlorophyll a content versus UTC time (dive No. 3, ARK 27/2).

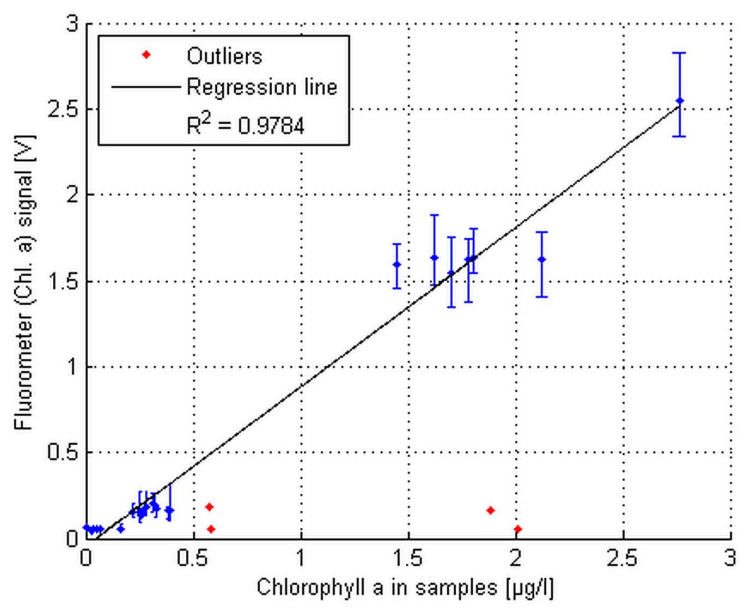

Fig. 7: Chlorophyll a calibration curve. The error bars represent the fluorometer values within $+/-5$ seconds around the time the sample was taken. The blue dots represent the average values of these intervals.

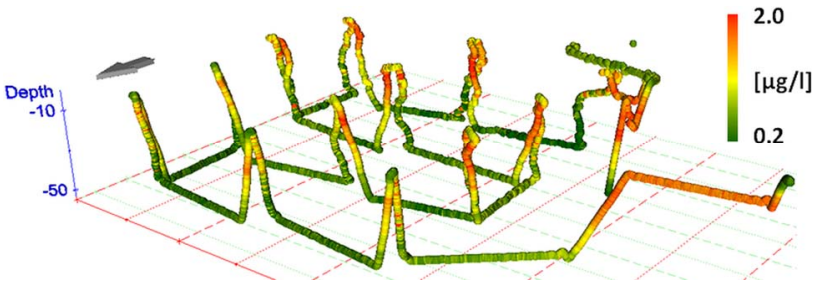

Fig. 8: Chlorophyll a distribution $[\mu \mathrm{g} / 1]$, Float phase, dive No. 3.

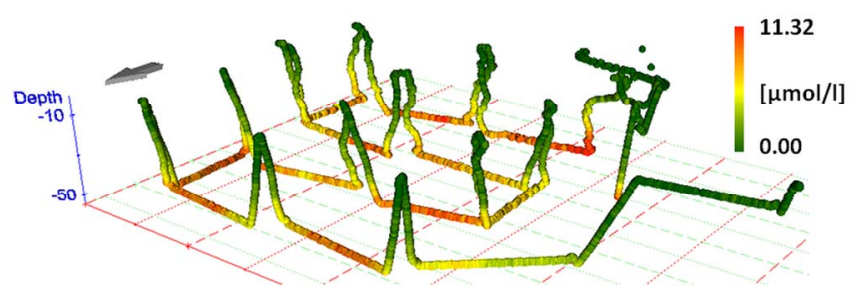

Fig. 9: Nitrate distribution [ $\mu \mathrm{mol} / 1]$, Float phase, dive No. 3.

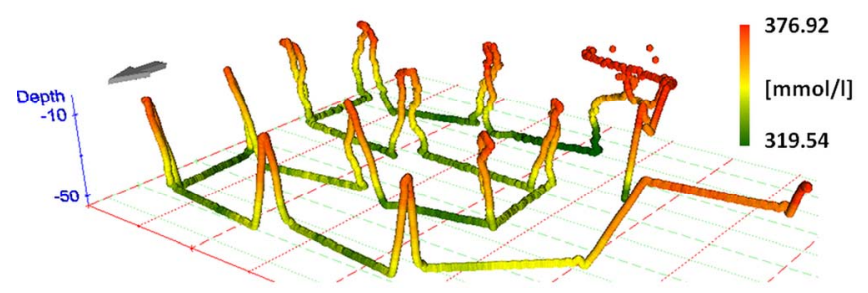

Fig. 10: Dissolved Oxygen [mmol/1], Float phase, dive No. 3.

\section{B. Under Ice}

The first two under ice dives, which PAUL accomplished in 2010 and 2011 respectively, were very similar (Fig. 11 and 12). As in the graphs before, the red circles indicate positions where the vehicle took water samples. Both missions were conducted at a constant depth of 27 meters (2010) and 20 meters (2011) and led the vehicle to a turning point about $2-2.5$ kilometers away from the ice edge. Both missions were accomplished under fast moving sea ice with a drift velocity of $1-1.5$ kilometers per hour. The evolutions of the ice fields were observed using satellite images days before the dive. During both dives a horizontal light profile, which shall exemplarily be shown here, was obtained using the PAR sensor. Although the inbound and the outbound tracks are located close to each other, there is a time mismatch in between them. As the ice moves during the dive it makes no sense to merge the PAR data of the inbound and outbound track into one single profile. Instead of that, Fig. 13 and 14 exclusively show the light profiles of the respective inbound track.

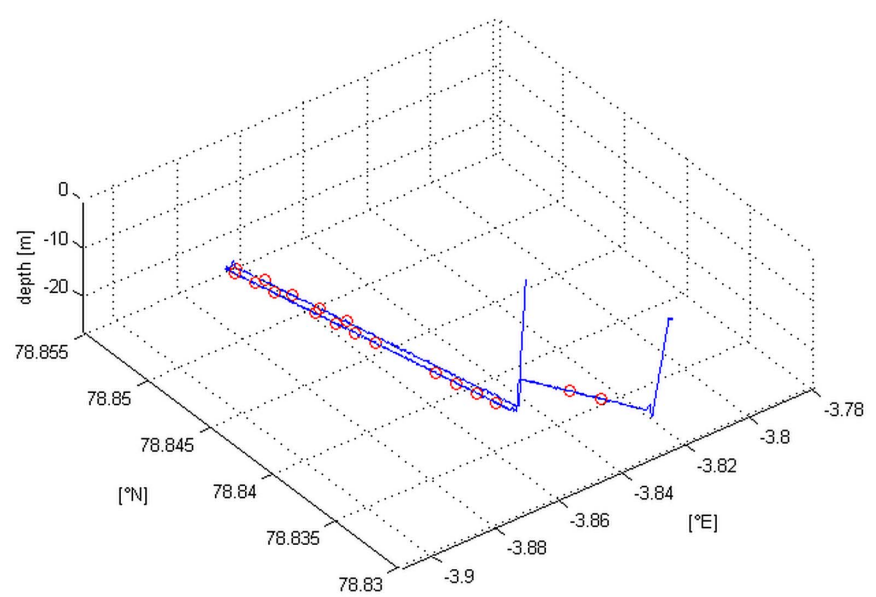

Fig. 11: Track of the very first under ice dive in 2010. After a short time at a depth of 20 meters the vehicle descended to the actual mission depth of 27 meters.

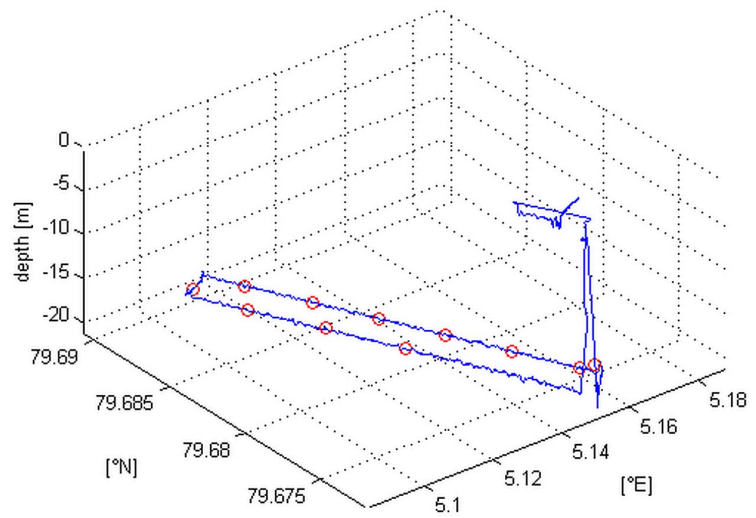

Fig. 12: Track of the under ice dive in 2011. The dive had to be aborted after half of the intended mission time. Thus the number of samples gathered during this dive is relatively small. 


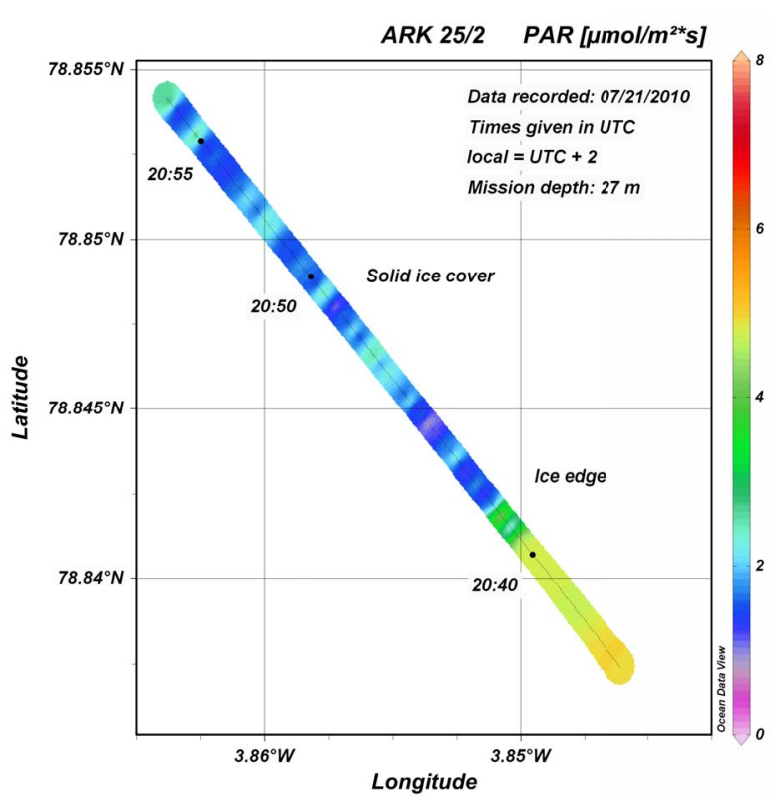

Fig. 13: Horizontal light profile (PAR) of the 2010 under ice dive.

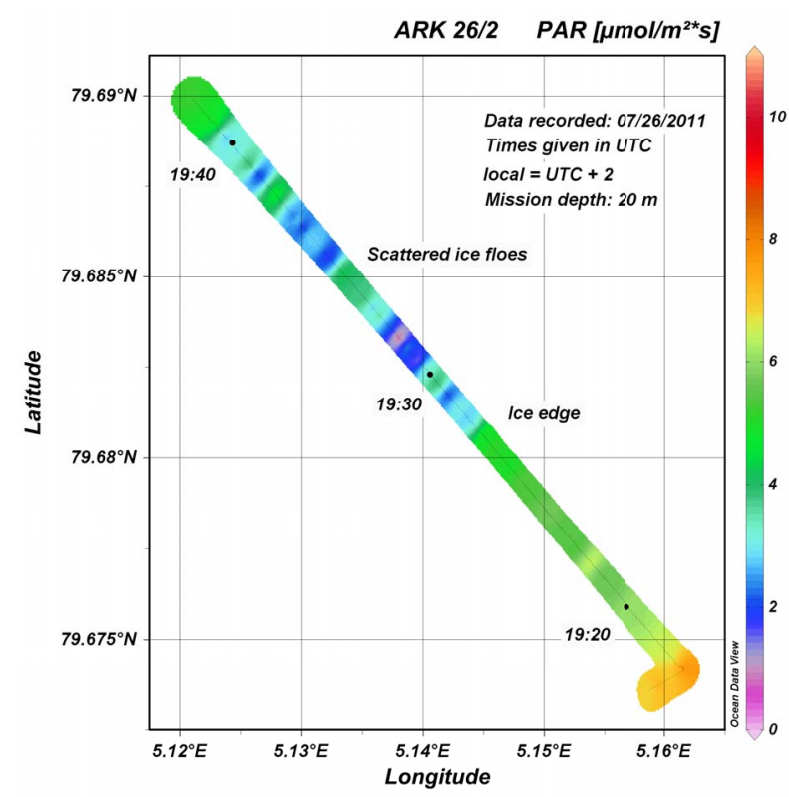

Fig. 14: Horizontal light profile (PAR) of the 2011 under ice dive. Notice the different PAR values below a solid ice cover and below a field of scattered ice floes.

In the framework of the Arctic expedition ARK 27/2 the Float maneuver was executed under ice for the first time. During dive 4A (07/24/2012) the AUV started the Floats at a depth of 50 meters and it approached the ice by a minimum distance of 10 meters. In Fig. 15 the red and black lines indicates the positions of the ice edge at the beginning of the dive (red) and at the end of the dive (black), respectively. The positions were determined using the GPS tracking system mentioned in chapter IV. Unfortunately, the power supply of the payload failed at the

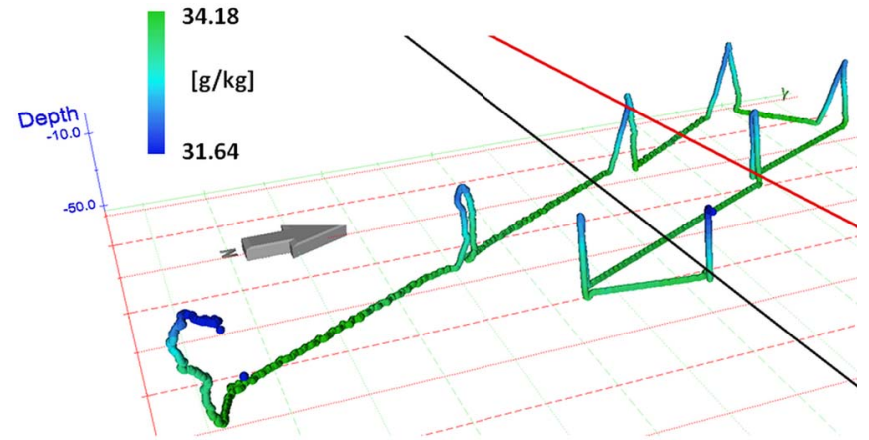

Fig. 15: Absolute salinity along the track of dive 4A. Notice the reduced salinity due to fresh water layers as the vehicle approaches the ice.

end of the dive, causing a severe data loss in the PCC. However, the data of the vehicle's CTD (Salinity is shown in Fig. 15) and the position data of the water samples could be saved.

\section{DISCUSSION}

The first operations using the Float maneuver showed encouraging results. As no dynamic problems occurred and as the maneuver, to the current state of knowledge, does not significantly influence the vehicle's navigation system, the Float maneuver can be expected to be used in future cruises as well. It has to be mentioned that all Floats which have been conducted to the present day, took approximately 4 to 4.5 minutes in average. The Float maneuvers started in 50 meters depth and ended close to the surface. This might be sufficient to investigate the upper water column. Yet, it has not been analyzed how longer Float phases influence the vehicle's navigation system.

Especially while the vehicle is slowly ascending from the Float maneuver, it delivers high resolution data with an accurate depth stamp. However, the quality of the data can be further improved. Of particular interest are the data of the $\mathrm{CO}_{2}$ and nitrate sensor. In the case of the $\mathrm{CO}_{2}$ sensor the correction method proposed by Fiedler et al in [25] is to be implemented in the future. In the case of the nitrate sensor the transmission of the sensor data via an analog signal reduces the data quality. As the analog signal does not contain any information about the recorded absorption spectra, the correction methods for bromide ions and CDOM (Sakamoto et al. [12], Zielinski et al. [26]) are currently not applicable. Although the SUNA nitrate sensor offers a special sea water mode, which makes the sensor using internal proxies to estimate bromide induced errors, there are some uncertainties remaining in the signal.

The data loss of the two under ice dives 4A and 4B during expedition ARK 27/2 is tragic as these dives represented one rare chance to combine physical and biochemical measurements from below the ice. Nevertheless, these dives demonstrated the enormous benefit marine research can derive from these kinds of AUV operations. Three dimensional imaging of the sensor data 
offers the possibility to quickly recognize correlations. As a final step of data imaging, there is currently work on software tools to interpolate in between the Floats and thus representing the data not just three dimensional, but volumetrically. This visualization method will provide the most intuitive impressions of what really happens in the oceans' uppermost meters.

\section{ACKNOWLEDGMENT}

In the past four years the AUV project was supported by a large number of people. The contribution of Antje Boetius, Oliver Zielinski and Frank Wenzhöfer should be particularly highlighted here as they decisively influenced the scientific orientation of the AUV project. Also, the authors would like to specially thank Uwe Wulff, a retired mathematician who set up an entirely new basis for AUV data processing and management. We thank Bluefin Robotics for their repeated support and especially Will O'Halloran who initially came up with the idea of a floating vehicle. The project has largely profited from the contribution and help of Jonas Hagemann, Thomas Soltwedel, Normen Lochthofen, Ilka Peeken, Michael Schlüter, Roi Martinez, Torben Gentz, Jana Hölscher, Kristin Hardge, Adam Mara, Kai-Uwe Ludwichowski and the professional support of the crews of RV Heincke and RV Polarstern.

Part of the scientific payload was developed with the financial support of the European Commission's 7th Framework Program (Research Infrastructures) "EUROFLEETS" (Contract No. 228344). Additionally, the financial contribution of the Institut français de recherche pour l'exploitation de la mer (Ifremer) for the development of the water sample collector is gratefully acknowledged.

\section{REFERENCES}

[1] W.O. Smith Jr., M.E.M. Baumann, D.V. Wilson, L. Aletsee, "Phytoplankton biomass and productivity in the marginal ice zone of the Fram Strait during summer 1984," Journal of Geophysical Research, Vol. 92, No. C7, pp. 6777-6786, 1987

[2] H.J. Niebauer and W.O. Smith Jr., "A numerical model of mesoscale physical-biological interactions in the Fram Strait marginal ice zone," Journal of Geophysical Research, Vol. 94, No. C11, pp. 16151-16175, 1989

[3] K.R. Arrigo, G. van Dijken, S. Pabi, "Impact of a shrinking ice cover on marine primary production," Geophysical Research Letters, Vol. 35, LI9603, 2008

[4] A.S. Brierly, P.G. Fernandes, M.A. Brandon, F. Armstrong, N.W. Millard, S.D. McPhail, P.Stevenson, M. Pebody, J. Perrett, M. Squires, D.G. Bone, G. Griffiths, "Antarctic Krill under sea ice: Elevated abundance in a narrow band just south of ice edge," Science, Vol. 295, No. 5561, pp. 1890-1892, 2002

[5] S.D. McPhail, M.E. Furlong, M. Pebody, J.R. Perrett, P. Stevenson, A. Webb, D. White, "Exploring beneath the PIG ice shelf with the Autosub3 AUV," Oceans 2009 - Europe, Conference Publication, 2009

[6] R.G. Perkin, E.L. Lewis, "Mixing in the West Spitsbergen Current," Journal of Physical Oceanography, Vol. 14, No. 8, pp. 1315-1325, 1984
[7] K.S. Johnson, L.J. Coletti, "In situ unltraviolet spectrophotometry for high resolution and long-term monitoring of nitrate, bromide and bisulfide in the ocean," Deep-Sea Research, Vol. 49, No. 7, pp. 1291-1305, 2002

[8] L.E. Bird, A. Sherman, J. Ryan, "Development of an active, large volume, discrete seawater sampler for autonomous underwater vehicles," Oceans 2007, Conference Publication, 2007

[9] T. Wulff, S. Lehmenhecker, U. Hoge, "Development and operation of an AUV-based water sample collector," Sea Technology, Vol. 51, No. 12, pp. $15-19,2010$

[10] Sea Bird Electronics Application Note 64 (July 2012)

[11] O. Zielinski, B. Fiedler, R. Heuermann, A. Körtzinger, E. Kopiske, G. Meinecke, K. Munderloh, "A new nitrate continous observation sensor for autonomous sub-surface applications: Technical design and first results," Oceans 2007 - Europe, Conference Publication, 2007

[12] C.M. Sakamoto, K.S. Johnson, L.J. Coletti, "Improved algorithm for the computation of nitrate concentrations in seawater using an in situ ultraviolet spectrophotometer," Limnology and Oceanography: Methods 7, Jan. 2009, pp. 132-143, 2009

[13] R.E. Zeebe and D. Wolf-Gladrow, " $\mathrm{CO}_{2}$ in seawater: equilibrium, kinetics, isotopes," Elsevier Oceanography Series, 65, p. 4, 2001

[14] J.B. SooHoo, D.A. Kiefer, D.J. Collins, I.S. McDermid, "In vivo fluorescence and absorption spectra of marine phytoplankton: I. taxonomic characteristics and responses to photoadaption," Journal of Plankton Research, Vol. 8, No. 1, pp. 197-214, 1986

[15] C. Büchel, C. Wilhelm, "In vivo analysis of slow chlorophyll fluorescence induction kinetics in algae: progress, problems and perspectives," Photochemistry and Photobiology, Vol. 58, No. 1, pp. 137-148, 1993

[16] A. Earp, C.E. Hanson, P.J. Ralph, V.E. Brando, S. Allen, M. Baird, L. Clementson, P. Daniel, A.G. Dekker, P.R.C.S. Fearns, J. Parslow, P.G. Strutton, P.A. Thompson, M. Underwood, S. Weeks, M.A. Doblin, "Review of fluorescent standards for calibration of in situ fluorometers: recommendations applied in coastal and ocean observing programs," Optics Express, Vol. 19, No. 27, pp. 26768-26782, 2011

[17] IXSEA GAPS Carbon V1 User Guide (January 2010), p. 11

[18] S. Lehmenhecker and T. Wulff, "Flying drone for AUV under-ice missions," Sea Technology, Vol. 54, No. 2, pp. 61-64, 2013

[19] T.J. Cowles, R.A. Desiderio, "Resolution of biological microstructure through in situ fluoresecence emission data: an oceanographic application using optical fibres," Oceanography, Vol. 6, No. 3, pp. 105-111, 1993

[20] M.M. Dekshenieks, P.L. Donaghay, J.M. Sullivan, J.E.B. Rines, T.R Osborn, M.S. Twardowski, "Temporal and spatial occurrence of thin phytoplankton layers in relation to physical processes," Marine Ecology Progress Series, Vol. 223, pp. 61-71, 2001

[21] J.P. Ryan, M.A. McManus, J.D. Paduan, F.P. Chavez, "Phytoplankton thin layers caused by shear in frontal zones of a coastal upwelling system," Marine Ecology Progress Series, Vol. 354, pp. 21-34, 2008

[22] R.Y. Tsai and R.K. Lenz, "A new technique for fully autonomous and efficient 3D robotics hand/eye calibration," IEEE Transactions on Robotics and Automation, Vol. 5, No.3, pp. 345-358, 1989

[23] J.T.O. Kirk, "Light and photosynthesis in aquatic ecosystems," $2^{\text {nd }}$ Edition., p. 113, 1994, ISBN 0521453534

[24] T. Suresh, M. Talaulikar, E. Desa, S.G.P. Matondkar, T.S. Kumar, A Lotlikar, "A simple method to minimize orientation effects in a profiling radiometer," Marine Geodesy, Vol. 35, No. 4, pp. 441-454, 2012

[25] B. Fiedler, P.Fietzek, N. Vieira, P. Silva, H.C. Bittig, A. Körtzinger, "In situ $\mathrm{CO}_{2}$ and $\mathrm{O}_{2}$ measurements on a profiling float," Journal of Atmospheric and Oceaninc Technology, Vol. 30, pp. 112-126, 2013

[26] O. Zielinski, D. Voß, B. Saworski, B. Fiedler, A. Körtzinger, "Computation of nitrate concentrations in turbid coastal waters using an in situ ultraviolet spectrophotometer," Journal of Sea Research, Vol. 65, No. 4, pp. 456-460, 2011 